\title{
Aporte de serapilheira e nutrientes ao solo em povoamentos jovens de Eucalyptus no planalto catarinense $^{1}$
}

\author{
Cíntia Neves Urbano ${ }^{2}$; Marcia Aparecida Simonete ${ }^{3}$; Paulo Roberto Ernani ${ }^{4}$; Djalma Miler Chaves ${ }^{5}$; \\ Letícia Moro ${ }^{6}$
}

Resumo: A deposição e decomposição de serapilheira em ecossistemas florestais pode variar com a idade e no decorrer do ano. Este trabalho tem por objetivo avaliar a deposição de serapilheira e quantificar a transferência de nutrientes ao solo ao longo do ano em povoamentos jovens de Eucalyptus dunnii Maiden. O estudo foi conduzido em Bocaina do Sul, SC, sobre um Cambissolo Húmico. Os tratamentos consistiram de avaliações em povoamentos de um, dois e três anos de idade. Em cada povoamento foram instaladas três parcelas e realizadas coletas mensais da serapilheira, utilizando 11 coletores de 0,25 $\mathrm{m}^{2}$ em cada parcela. Foi quantificada a deposição de matéria seca de cada componente da serapilheira (folhas, ramos e casca), e determinado os teores de $\mathrm{P}, \mathrm{S}, \mathrm{K}, \mathrm{Ca}$ e $\mathrm{Mg}$. Os três povoamentos apresentaram deposições anuais semelhantes de serapilheira, sendo cerca de $95 \%$ da biomassa representado pelas folhas. A deposição de serapilheira foi sazonal, com menor deposição no inverno e maior no verão. As folhas foram o principal contribuinte na transferência de nutrientes, transferindo em média $99 \%$ do P, $97 \%$ de K, $97 \%$ de Ca, $96 \%$ de $\mathrm{Mg}$ e $98 \%$ de S. A magnitude de transferência de foi: $\mathrm{Ca}>\mathrm{K}>\mathrm{Mg}>\mathrm{S}$ $>$ P.

Palavras - chave: Componentes da serapilheira; Ciclagem de nutrientes; Silvicultura.

\section{Litter fall production and nutrient adition by young plantations of Eucalyptus in the southern plateau of Santa Catarina, Brazil}

\begin{abstract}
Litter deposition and decomposition of litter may vary with age and, during the year, with climatic seasons. The objective of this study was to quantify the litter produced and the transfer of nutrients to the soil throughout the year in young stands of Eucalyptus dunnii Maiden. Field experiments were carried out over an Haplumbrept, in Bocaina do Sul, Santa Catarina State, Southern Brazil, with one, two and three year-old stands Three plots were installed int each plantation age, in order to collect plant litter. Litter sampling was performed monthly, during the entire year of 2011. It was used 11 collectors of $0.25 \mathrm{~m}^{2}$ in each plot. It was quantified the deposition of dry matter of each component of litter (leaves, branches and bark) over the seasons, and determined the levels of $\mathrm{P}, \mathrm{S}, \mathrm{K}, \mathrm{Ca}$ and. The three stands showed similar annual litter depositions, and about $95 \%$ of the total was constituted by leaves. Litter deposition presented seasonality, with the lowest deposition in the winter and the highest in the summer. Litter deposition was seasonal, with lower deposits in winter and higher in summer 99\% P, $97 \% \mathrm{~K}, 97 \% \mathrm{Ca}, 96 \% \mathrm{Mg}$ and $98 \% \mathrm{~S}$. The magnitude of values was: $\mathrm{Ca}>\mathrm{K}>\mathrm{Mg}>\mathrm{S}>\mathrm{P}$.
\end{abstract}

Keywords: Litter components; Nutrient cycling; Silviculture.

\footnotetext{
${ }^{1}$ Recebido em 09.05.2017 e aceito para publicação como artigo científico em 23.08.2018.

${ }^{2}$ Engenheira Agrônoma, Responsável Técnica na Empresa Yara Fertilizantes. E-mail: <cintia.neves@yara.com>

${ }^{3}$ Engenheira Agrônoma, Dra., Responsável Técnica na Empresa Blumeterra Serviços e Comércio Ltda. E-mail: <marciasimonete@gmail.com>

${ }^{4}$ Engenheiro Agrônomo, Dr., Professor Titular da Universidade do Estado de Santa Catarina (UDESC), Lages. E-mail: <paulorobertoernani@gmail.com>

${ }^{5}$ Engenheiro Agrônomo, Dr., Consultor da Empresa Klabin S.A. E-mail: <dm.chaves@terra.com.br >

${ }^{6}$ Engenheira Florestal, Dra. Professora do Centro de Educação Profissional Caetano Costa/CEDUP. E-mail:

< leticia_moro@hotmail.com>
} 


\section{Introdução}

No Planalto Catarinense, grandes áreas vêm sendo ocupadas com E. dunnii e E. benthamii para a produção de celulose, tendo em vista sua resistência à geada, muito comum na região durante os meses de inverno. Estas espécies são de rotação curta (6 a 7 anos), de alta produtividade (FOELKEL, 2015) e requerem grande quantidade de nutrientes, principalmente nos anos iniciais de crescimento (LACLAU et al., 2010), o que torna a manutenção do processo de ciclagem de nutrientes importante para a sustentabilidade dos povoamentos.

A serapilheira constitui o material vegetal depositado ao solo pelas árvores, como: folhas, galhos, e miscelânea, que compreende as estruturas reprodutivas, como flores, frutos e sementes. A importância desse material na ciclagem de nutrientes é evidenciada de forma mais clara em florestas que se mantêm em solos de baixa fertilidade (VEZZANI e MIELNICZUK, 2011).

A quantificação dos nutrientes da serapilheira, bem como o padrão de sua ciclagem, torna possível a quantificação do aporte e das saídas ou perdas de nutrientes do ecossistema florestal (VITOUSEK, 1984). Esse tipo de estudo possibilita uma avaliação mais abrangente das implicações nutricionais da exploração florestal no potencial produtivo (LACLAU et al., 2010), reduzindo o déficit de nutrientes no solo (SCHUMACHER et al., 2008) e possibilitando a produção de sucessivas rotações (VIERA e SCHUMACHER, 2010).

Segundo Fortes et al. (2008) a deposição de serapilheira em ecossistemas florestais é variável no decorrer do ano e se encontra sob determinação das estações climáticas. Entre os fatores que afetam a quantidade de produção de serapilheira em ecossistemas florestais, Correia e Andrade (1999) relatam que os mais relevantes são as variáveis climáticas relacionados com precipitação pluviométrica e temperatura. Em estudo com Eucalyptus na região Sul do Brasil, Corrêa et al. (2013) e Inkotte et al. (2015) verificaram maior deposição de serapilheira em resposta direta ao aumento de temperatura. Barlow et al. (2007) encontraram correlação positiva entre a deposição mensal de folhas de $E$. urophylla com 4 a 5 anos de idade e a precipitação mensal em clima com estação seca na Amazônia, sendo que a decomposição da serapilheira ocorre com maior rapidez nos locais onde ocorrem as maiores precipitações pluviométricas (SELLE, 2007).

Diante do exposto, a realização de trabalhos nas regiões subtropicais é de fundamental importância para que se tenham conhecimentos acerca da dinâmica da produção de serapilheira e do teor de nutrientes contidos nos seus diferentes componentes (folhas, galhos, cascas e outras partes das plantas) e para que se possa adotar um manejo que venha assegurar a sustentabilidade dos povoamentos de Eucalyptus.

Este trabalho teve por objetivo avaliar a produção de serapilheira e quantificar a transferência anual de nutrientes das copas das árvores ao solo durante as diferentes estações do ano, em povoamentos de Eucalyptus dunnii com um, dois e três anos de idade.

\section{Material e métodos}

O estudo foi realizado na Fazenda Guarujá, de propriedade da Klabin S.A., no município de Bocaina do Sul, SC, a uma altitude média de 877 $\mathrm{m}$, nas seguintes coordenadas: $27^{\circ} 41^{\prime} 4^{\prime \prime} \mathrm{S}$ e $49^{\circ}$ $58^{\prime} 41^{\prime \prime} \mathrm{O} ; 27^{\circ} 40^{\prime} 43.55^{\prime \prime} \mathrm{S}$ e $49^{\circ} 59^{\prime} 15^{\prime \prime} \mathrm{O} ; 27^{\circ}$ $41^{\prime} 22^{\prime \prime} \mathrm{S}$ e $49^{\circ} 59^{\prime} 25^{\prime \prime} \mathrm{O}$. Nela, foram plantados povoamentos de Eucalyptus dunnii Maiden, em novembro de 2009, novembro de 2008 e dezembro de 2007, respectivamente, cujos anos de plantio constituíram os tratamentos.

O clima do local é classificado como mesotérmico úmido, $\mathrm{Cfb}$ na Classificação Climática de Köppen, com verões frescos e chuvas bem distribuídas durante 0 ano (Embrapa, 2004). O solo da área experimental é classificado como Cambissolo Húmico Distrófico típico (SANTOS et al., 2006), cujas principais características são fornecidas na Tabela 1. 
Tabela 1 - Características do Cambissolo Húmico nas áreas experimentais de cultivo de Eucalyptus dunnii de um, dois e três anos de idade ${ }^{1}$.

Table 1 - Characteristics Haplumbrept in the experimental sites with one, two and three year old Eucalyptus dunnii plantations.

\begin{tabular}{|c|c|c|c|c|c|c|c|c|c|}
\hline \multirow{2}{*}{$\begin{array}{c}\text { Profundidade } \\
(\mathrm{cm})\end{array}$} & \multirow[t]{2}{*}{$\mathrm{pH}_{\text {água }}$} & Argila & $\mathrm{MO}$ & \multirow{2}{*}{\multicolumn{2}{|c|}{$\left(\mathrm{mg} \mathrm{dm}^{-3}\right)$}} & $\mathrm{Ca}$ & $\mathrm{Mg}$ & $\mathrm{Al}$ & CTC \\
\hline & & \multicolumn{2}{|c|}{$(\%)$} & & & \multicolumn{4}{|c|}{$\left(\mathrm{cmol}_{c} \mathrm{dm}^{-3}\right)$} \\
\hline \multicolumn{10}{|c|}{ Povoamento de 1 ano } \\
\hline $0-20$ & 4,2 & 25 & 4,4 & 6,8 & 76 & 0,94 & 0,66 & 9,34 & 11,13 \\
\hline $20-40$ & 4,3 & 30 & 2,6 & 5,7 & 55 & 0,47 & 0,14 & 9,32 & 10,07 \\
\hline \multicolumn{10}{|c|}{ Povoamento de 2 anos } \\
\hline $0-20$ & 4,1 & 20 & 3,1 & 12,2 & 88 & 0,72 & 0,29 & 10,7 & 11,90 \\
\hline $20-40$ & 4,0 & 27 & 2,2 & 6,1 & 81 & 0,52 & 0,19 & 11,2 & 12,10 \\
\hline \multicolumn{10}{|c|}{ Povoamento de 3 anos } \\
\hline $0-20$ & 3,8 & 18 & 3,9 & 10,6 & 33 & 0,57 & 0,12 & 10,8 & 11,59 \\
\hline $20-40$ & 4,0 & 21 & 3,5 & 5,6 & 29 & 0,37 & 0,08 & 10,0 & 10,53 \\
\hline
\end{tabular}

A área experimental se encontra no segundo ciclo de cultivo com espécies florestais, tendo sido cultivada anteriormente com Pinus taeda, cujas árvores foram cortadas aos 17 anos de idade. Os povoamentos de um, dois e três anos de Eucalyptus dunnii Maiden, foram implantados com espaçamento de 2,5 $\mathrm{m}$ x 2,5 m. Antes de cada plantio, foi realizada subsolagem, a uma profundidade média de $45 \mathrm{~cm}$, quando foram aplicados $200 \mathrm{~kg} \mathrm{ha}^{-1}$ de fosfato natural reativo (Gafsa).

As mudas foram fertilizadas aos 15 dias após o plantio, com $150 \mathrm{~g}$ por planta do adubo da fórmula 06-30-06 (N-P-K, nas formas de ureia, superfosfato triplo e cloreto de potássio, respectivamente). $\mathrm{O}$ fertilizante foi aplicado em duas pequenas covas laterais, de aproximadamente $5 \mathrm{~cm}$ de diâmetro, a $10 \mathrm{~cm}$ de profundidade e a $10 \mathrm{~cm}$ distante da planta, sendo aplicado metade da dose em cada cova. Aos três e aos seis meses após cada plantio foram realizadas adubações de cobertura com $200 \mathrm{~g}$ do adubo da fórmula 15-05-30 (N-P-K, nas formas de ureia, superfosfato triplo e cloreto de potássio, respectivamente), na superfície do solo, na área de projeção da copa de cada árvore. O controle das plantas espontâneas foi feito por meio do uso do herbicida pós-emergente (glifosato), e o controle de formigas foi efetuado com iscas granuladas (sulfluramida).

Em cada um dos três povoamentos foram instaladas três parcelas de $20 \mathrm{~m}$ x $30 \mathrm{~m}$, nas quais foram dispostos 11 coletores, a uma altura de 50 $\mathrm{cm}$ do solo e distribuídos na parcela em formato de " $x$ ". Os coletores tinham dimensões de $50 \mathrm{~cm}$ x $50 \mathrm{~cm}$ x $10 \mathrm{~cm}$, com moldura de madeira e fundo de tela de nylon com malha de $2 \mathrm{~mm}$. A cada 30 dias, durante um ano (de dezembro de 2010 a novembro de 2011), a serapilheira depositada em cada um dos coletores foi recolhida, separada em três componentes, folhas, casca e galhos, os quais foram posteriormente secos em estufa de circulação forçada de ar, a 60 ${ }^{\circ} \mathrm{C}$, até peso constante. $\mathrm{Na}$ sequência, foi quantificada a massa de matéria seca mensal de cada componente e calculadas as quantidades totais de folhas, cascas e galhos obtidos em cada estação climática, assim como o total de serapilheira. Os diferentes componentes da serapilheira coletados mensalmente (folhas, casca e galhos) foram separadamente moídos e submetidos a análise, após digestão nitroperclórica, para determinação dos teores de fósforo, potássio, cálcio, magnésio e enxofre, conforme metodologia descrita por Tedesco et al. (1995). De posse dos dados mensais da massa seca e do teor de nutrientes de cada componente da serapilheira, foi estimado, por meio de média ponderada, o aporte total dos respectivos nutrientes para cada estação do ano.

Para auxiliar na interpretação dos resultados obtidos, foram utilizados dados meteorológicos 
de temperatura média estacional (calculada pela média das temperaturas mínimas e máximas diárias e mensais) e de precipitação pluviométrica média estacional (calculada pela precipitação diária e mensal). Esses dados foram coletados na estação meteorológica da Empresa de Pesquisa Agropecuária e Extensão Rural de Santa Catarina (EPAGRI, 2012), localizada no município de Lages, com altitude de $938 \mathrm{~m}$, cujas coordenadas geográficas são $27^{\circ} 48^{\prime} 27^{\prime \prime} \mathrm{S}$ de latitude e $50^{\circ} 19^{\prime}$ 44". A distância entre a estação meteorológica e as áreas experimentais eram de aproximadamente $37,22 \mathrm{~km}, 36,54 \mathrm{~km}$ e $35,89 \mathrm{~km}$, para os respectivos povoamentos de um, dois e três anos.

Os dados de biomassa e teor de nutrientes dos diferentes componentes da serapilheira foram analisados estatisticamente segundo o delineamento de blocos ao acaso, com três repetições. Foi realizada a análise de variância (ANOVA) utilizando o aplicativo "Sisvar" (FERREIRA, 2003). Quando houve significância estatística, procedeu-se à comparação das médias por meio do teste Tukey a 5\%. Para avaliar o efeito da temperatura e da precipitação estacional sobre a produção de serapilheira, os dados foram submetidos à análise de correlação linear pelo teste $t$ ao nível de $5 \%$.

\section{Resultados e Discussão}

A deposição de serapilheira nos três povoamentos apresentou variação ao longo das estações climáticas durante $\mathrm{o}$ período de avaliação (Tabela 2). Considerando os três povoamentos, em geral, a maior deposição da fração folha da serapilheira ocorreu no verão e na primavera, enquanto que a deposição de casca aconteceu no outono e no inverno. Por outro lado, a deposição de galho foi pequena e variou ao longo das estações. A fração folha foi a que apresentou maior deposição em todas as estações, seguidas do galho e casca.

A deposição anual de serapilheira (folha + galho + casca) nos povoamentos de E. dunnii não diferiram significativamente, produzindo em média cerca de $6.560 \mathrm{~kg} \mathrm{ha}^{-1} \mathrm{ano}^{-1}$ (Tabela 2).

Tabela 2 - Deposição de cada componente da serapilheira (folha, galho e casca) ao longo das estações do ano, em povoamentos de Eucalyptus dunnii com diferentes idades.

Table 2 - Sazonal response of litterfall component deposition in Eucalyptus dunnii stands of different ages.

\begin{tabular}{|c|c|c|c|c|c|c|c|c|c|}
\hline \multirow{3}{*}{$\begin{array}{l}\text { Estações } \\
\text { do ano }{ }^{1} \\
\end{array}$} & \multicolumn{9}{|c|}{ Deposição de serapilheira por componente $\left(\mathrm{kg} \mathrm{ha}^{-1}\right)^{2}$} \\
\hline & \multicolumn{3}{|c|}{1 ano de idade } & \multicolumn{3}{|c|}{2 anos de idade } & \multicolumn{3}{|c|}{3 anos de idade } \\
\hline & Folha & Galho & Casca & Folha & Galho & Casca & Folha & Galho & Casca \\
\hline Verão & $\begin{array}{l}2141 \mathrm{Aa} \\
(81,41)^{3 ; 4}\end{array}$ & $\begin{array}{c}3 \text { Aa } \\
(0,50)\end{array}$ & $\begin{array}{l}0 \mathrm{Bab} \\
(0,00)\end{array}$ & $\begin{array}{c}2236 \mathrm{Aa} \\
(42,34)\end{array}$ & $\begin{array}{l}21 \mathrm{Bab} \\
(3,21)\end{array}$ & $\begin{array}{c}1 \mathrm{Ba} \\
(0,20)\end{array}$ & $\begin{array}{c}2192 \mathrm{Aa} \\
(55,74)\end{array}$ & $\begin{array}{l}26 \mathrm{Ba} \\
(1,30)\end{array}$ & $\begin{array}{l}32 \mathrm{Ba} \\
(2,49)\end{array}$ \\
\hline Outono & $\begin{array}{c}1392 \mathrm{ABa} \\
(37,91)\end{array}$ & $\begin{array}{l}37 \mathrm{Aa} \\
(1,37)\end{array}$ & $\begin{array}{c}8 \mathrm{Ab} \\
(0,48)\end{array}$ & $\begin{array}{c}1639 \text { Aba } \\
(44,85)\end{array}$ & $\begin{array}{c}44 \mathrm{ABa} \\
(4,21)\end{array}$ & $\begin{array}{l}74 \mathrm{Aa} \\
(5,70)\end{array}$ & $\begin{array}{c}1159 \mathrm{BCa} \\
(22,99)\end{array}$ & $\begin{array}{c}84 \mathrm{ABa} \\
(2,13)\end{array}$ & $\begin{array}{c}104 \mathrm{Aa} \\
(6,20)\end{array}$ \\
\hline Inverno & $\begin{array}{l}1113 \mathrm{Bab} \\
(40,74)\end{array}$ & $\begin{array}{l}26 \mathrm{Ab} \\
(7,51)\end{array}$ & $\begin{array}{l}11 \mathrm{Ab} \\
(0,79)\end{array}$ & $\begin{array}{c}969 \mathrm{Bab} \\
(32,50)\end{array}$ & $\begin{array}{l}109 \text { Aa } \\
(9,01)\end{array}$ & $\begin{array}{l}46 \mathrm{Aa} \\
(3,12)\end{array}$ & $\begin{array}{l}601 \mathrm{Ca} \\
(16,02)\end{array}$ & $\begin{array}{l}92 \mathrm{ABa} \\
(10,73)\end{array}$ & $\begin{array}{c}59 \mathrm{ABa} \\
(6,42)\end{array}$ \\
\hline Primavera & $\begin{array}{l}1431 \mathrm{ABb} \\
(55,32)\end{array}$ & $\begin{array}{l}33 \mathrm{Ab} \\
(4,32)\end{array}$ & $\begin{array}{c}1 \mathrm{Bb} \\
(0,37)\end{array}$ & $\begin{array}{l}2213 \mathrm{Aa} \\
(109,37)\end{array}$ & $\begin{array}{c}55 \mathrm{ABb} \\
(4,81)\end{array}$ & $\begin{array}{r}9 \mathrm{Ba} \\
(0,94)\end{array}$ & $\begin{array}{c}1580 \mathrm{ABb} \\
(87,31)\end{array}$ & $\begin{array}{l}123 \text { Aa } \\
(10,97)\end{array}$ & $\begin{array}{l}26 \mathrm{Ba} \\
(3,28)\end{array}$ \\
\hline \multirow[t]{2}{*}{ Total } & $\begin{array}{c}6077 \mathrm{~b} \\
(40,06)^{5}\end{array}$ & $\begin{array}{c}99 \mathrm{~b} \\
(2,34)\end{array}$ & $\begin{array}{c}20 \mathrm{c} \\
(0,33)\end{array}$ & $\begin{array}{l}7056 \mathrm{a} \\
(46,02)\end{array}$ & $\begin{array}{l}229 \mathrm{a} \\
(3,29)\end{array}$ & $\begin{array}{l}130 \mathrm{~b} \\
(2,14)\end{array}$ & $\begin{array}{l}5534 \mathrm{~b} \\
(43,57)\end{array}$ & $\begin{array}{l}325 \mathrm{a} \\
(4,47)\end{array}$ & $\begin{array}{l}221 \mathrm{a} \\
(2,87)\end{array}$ \\
\hline & \multicolumn{3}{|c|}{ Folha + galho + casca $=6196 a(26,43)^{6}$} & \multicolumn{3}{|c|}{ Folha + galho + casca $=7415 \mathrm{a}(30,26)$} & \multicolumn{3}{|c|}{ Folha + galho + casca $=6080 \mathrm{a}(24,78)$} \\
\hline
\end{tabular}


O componente constituído pelas folhas foi o que mais contribuiu, sendo a contribuição média na produção total de serapilheira, nos povoamentos de um, dois e três anos foram, respectivamente, de $98 \%, 95 \%$ e $91 \%$. Para componente galho, a contribuição foi intermediária $(1,6 \%, 3,2 \%$ e $5,4 \%$, respectivamente), enquanto que a fração casca foi a que menos contribuiu $(0,4 \%, 1,8 \%$ e $3,6 \%$, respectivamente). Deste modo, a contribuição dos componentes da serapilheira na deposição anual de serapilheira nos três povoamentos seguiu a ordem folha $>$ galho $>$ casca.

A contribuição da fração folha na deposição total da serapilheira observada para os três povoamentos de E. dunnii está de acordo com os estudos realizados em florestas subtropicais com esta espécie, os quais relatam uma maior contribuição das folhas ao total de serapilheira produzida (SCHUMACHER et al. 2013; CORRÊA et al., 2013; INKOTTE et al., 2015). Por outro lado, a pequena quantidade de deposição de galhos e casca, em relação a deposição de folhas da serapilheira, pode ser justificada por se tratar de povoamentos jovens, onde ainda não se iniciou de forma intensa os processos de desrama natural e de soltura de casca (na forma de ritidoma), os quais iniciam a partir dos 3 a 4 anos (FOELKEL, 2015).

As quantidades de folhas, casca e galhos depositados na serapilheira encontradas neste estudo são concordante com as encontradas por Corrêa et al. (2013) em povoamento jovem de E. dunnii avaliado entre 16,5 a 28,5 meses de idade no bioma Pampa, RS, onde a fração folha contribui com $93 \%$, miscelânea com $6 \%$ e galho grosso com $1 \%$ em relação à biomassa total.

Verificou-se, neste trabalho, que há uma tendência na redução da contribuição das folhas e um aumento na contribuição dos galhos e da casca na serapilheira com o aumento da idade dos povoamentos. Segundo Schumacher et al. (2011), as folhas normalmente constituem a maior proporção da biomassa de serapilheira que caem ao solo, aumentando com a idade, até que em certo estágio de desenvolvimento ocorre uma diminuição, em razão do aumento na queda de galhos e casca (REIS e BARROS, 1990), permanecendo entre 60 a $80 \%$ do total depositado para diferentes formações florestais (SCHUMACHER et al. 2013; CIZUNGU et al., 2014). Em povoamento de Eucalyptus urograndis (E. grandis $x$ E. urophylla) com 30 anos de idade, Diniz et al. (2011) observaram maior contribuição das folhas $(65 \%)$ e galhos (27\%), como componentes do material decíduo. Segundo Bray e Gohran (1964), a produção de serapilheira em diferentes florestas nativas estabilizadas é constituída por 60 a $80 \%$ de folhas, 1 a $15 \%$ de galhos, e 1 a $25 \%$ de cascas das árvores.

A deposição total de serapilheira, dentro de cada estação do ano, entre os povoamentos, foi estatisticamente semelhante na maioria das estações, sendo que apenas na primavera o povoamento de um ano apresentou menor deposição do que os outros dois (Figura 1). Verificou-se, entretanto, que em cada plantio a deposição total de serapilheira variou ao longo das estações do ano, sendo a menor produção observada na estação do inverno. Para os três povoamentos, a produção de serapilheira estacional apresentou coeficiente de correlação simples significativo com dados de temperatura, fato não observado com dados de precipitação. A maior produção de serapilheira foi verificada nos períodos de temperaturas mais elevada.

Quanto à produção total anual de serapilheira nos três povoamentos, a deposição de serapilheira foi de ordem próxima das encontradas por Cunha Neto et al. (2013), de $8830 \mathrm{~kg} \mathrm{ha}^{-1}$ ano $^{-1}$, com E. urograndis, com 3,5 a 4,5 anos de idade; Schumacher et al. (2013), de $7440 \mathrm{~kg} \mathrm{ha}^{-1} \mathrm{ano}^{-1}$, com E. urophylla $\times E$. globulus, com 5,6 a 7,6 anos de idade; e Silva et al. (2014), de $6500 \mathrm{~kg} \mathrm{ha}^{-1} \mathrm{ano}^{-1}$, com E. grandis, com 3 a 4 anos de idade. 


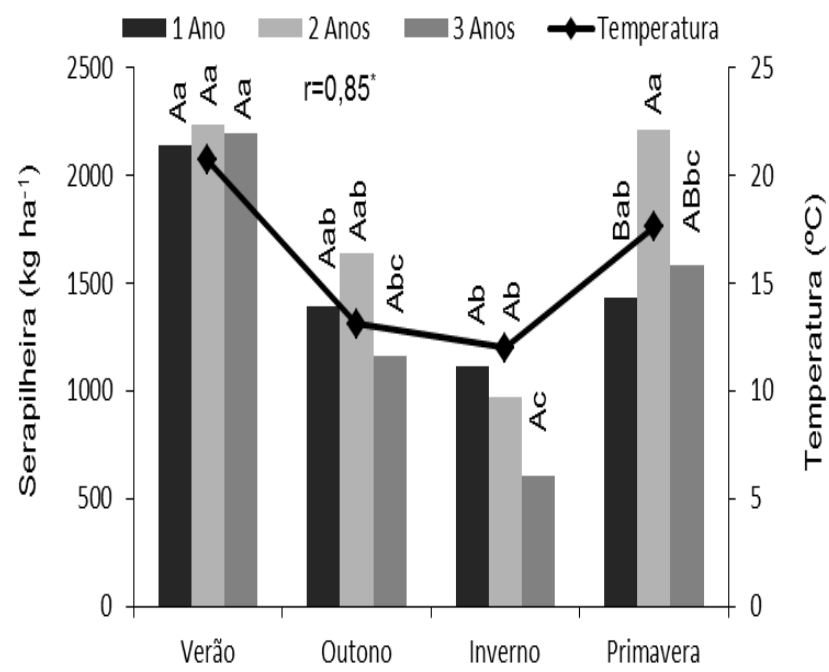

A

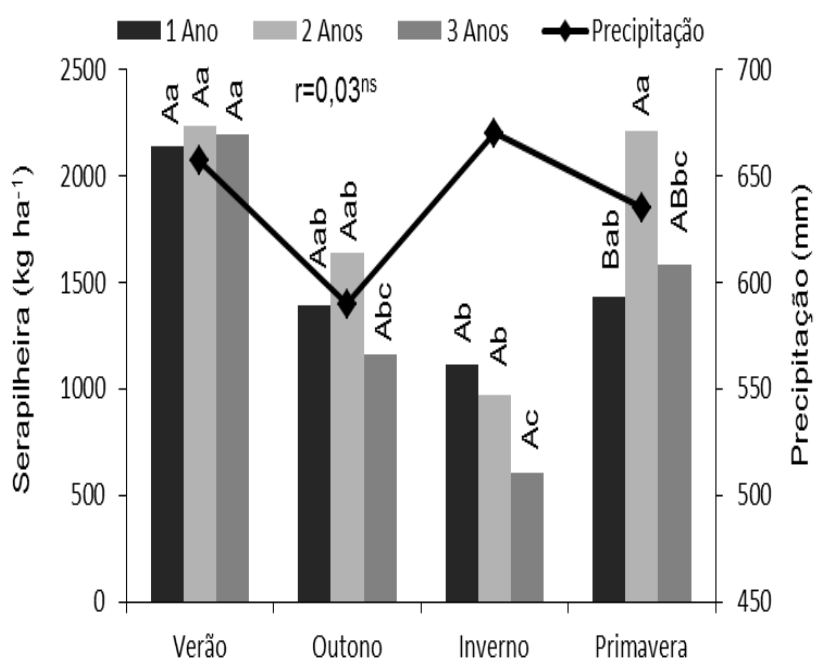

B

Figura 1 - Deposição total de serapilheira (folha + galho+ casca) comparada com variação média da temperatura (A) e precipitação (B) ao longo das estações do ano (dezembro de 2010 a novembro de 2011) em povoamentos de Eucalyptus dunnii. Médias seguidas de letras diferentes em maiúscula, que representam a comparação dentro de cada estação para povoamentos distintos, e minúsculas, que representam a comparação dentro de cada povoamento e entre estações, diferem entre si pelo teste de Tukey ao nível de 5\%. ${ }^{*}$, ns Essas indicam que as correlações entre deposição de serapilheira e temperatura (A) e precipitação (B) foram significativas e não significativas, respectivamente, pelo teste $t$ ao nível de 5\%.

Figure 1 - Sazonal total litterfall (leave + shoot + bark) compared to variation on mean temperature (A) and rainfall (B) from december 2010 to november 2011 in Eucalyptus dunnii stands. Means followed by different letters uppercase representing the comparison withi each station in stands of different ages and lowercase within each planting and between stations differ by Tukey test at $5 \% .{ }^{*}$, ns They indicate that the correlation between litter production and temperature (A) and precipitation (B) were significant and not significant, respectively, by t test at 5\%.

A correlação significativa da deposição de serapilheira apenas com a variável climática de temperatura demonstra que a sazonalidade climática observada no Planalto Catarinense se deve principalmente à variação de temperatura, uma vez que a precipitação mensal nas diferentes estações permanece acima de 100 $\mathrm{mm}$, assegurando um bom suprimento hídrico ao longo do ano (EPAGRI, 2012). De forma concordante, Corrêa et al. (2013), avaliando a produção de serapilheira em um povoamento de E. dunnii, em Alegrete, RS, observaram correlação significativa da produção de serapilheira apenas com dados de temperatura e não com a precipitação. Em função da distribuição de precipitação ser aproximadamente homogênea ao longo do ano e, ser evidenciado nas classificações climáticas do Estado do Rio Grande do Sul, como a de Maluf (2000), que utiliza como critério inicial variáveis de temperatura.
O padrão sazonal de produção de serapilheira verificado nesse trabalho é semelhante ao reportado por Viera et al. (2014) em povoamento de E. urophylla $\times$ E. globulus, em Eldorado do Sul-RS, onde foi observado, ao longo de 3 anos de estudo, maior deposição de serapilheira no período quente (primavera e verão). Sazonalidade na deposição de frações de serapilheira também foi encontrada por Salvador et al. (2014) ao estudarem a produção de serapilheira em um povoamento de E. grandis com 4,4 a 5,5 anos de idade, em São Gabriel-RS, onde a maior deposição de serapilheira ocorreu no período quente. Em estudo com E. benthamii, com 7 anos de idade, em Lages-SC, Inkotte et al. (2015) constataram maior deposição de serapilheira na primavera seguida do verão.

A maior deposição de serapilheira durante o período de elevação de temperatura (primavera e verão) observado neste estudo está associada ao maior crescimento vegetativo. Com a elevação 
da temperatura ocorre uma constante troca de folhas das árvores, proporcionada pela maior atividade fisiológica dos indivíduos neste período, fazendo com que ocorra uma intensa troca da folhagem, quando o material senescente é liberado, dando lugar a uma folhagem nova e fotossinteticamente mais ativa (SCHUMACHER et al., 2003). Além disso, com o aumento do volume de copa neste período ocorre o sombreamento mútuo dos ramos inferiores da copa promovendo a queda das folhas. Segundo Turner e Lambert (1983), o aumento na deposição de serapilheira se correlaciona positivamente com incrementos da biomassa foliar e da área basal das árvores. Em plantio de E. dunnii, localizado em Bagé-RS, cujo monitoramento seguiu de aproximadamente 1,4 a 2,4 anos de idade, o período de maior deposição de serapilheira ocorreu a partir do início do período de crescimento vegetativo e do fechamento do dossel (CORRÊA et al., 2013).

Para os teores de nutrientes nos diferentes componentes (folha, galho e casca) da serapilheira, os teores de $\mathrm{P}, \mathrm{K}, \mathrm{Mg}$ e $\mathrm{S}$ foram, em grande parte, menores na estação de verão, enquanto que para o $\mathrm{Ca}$ foi observado maior teor, em todos os povoamentos. O povoamento de três anos apresentou, em geral, menor teor de P, K, $\mathrm{Ca}$ e $\mathrm{S}$ nos componentes folha e galho, principalmente nas estações de outono e inverno, em relação aos povoamentos de um e dois anos. Para o Mg, o menor teor foi observado no povoamento de dois anos em praticamente todos os componentes (Tabela 3). Contudo, em geral, nos três povoamentos o teor de nutrientes seguiu a ordem folha > galho > casca, sendo que as folhas apresentaram maiores teores dos nutrientes, cuja ordem foi $\mathrm{Ca}>\mathrm{K}>\mathrm{Mg}>\mathrm{S}>\mathrm{P}$. A ordem de teor de nutrientes das folhas verificados nos povoamentos desse estudo estão de acordo com resultados encontrados por Hernández et al. (2009), Freitas et al. (2013), Ashagrie e Zech (2013) e Viera et al. (2014), que observaram maiores teores de $\mathrm{Ca}$, seguidos do $\mathrm{N}, \mathrm{K}, \mathrm{Mg}, \mathrm{S}$ e $\mathrm{P}$ em diferentes povoamentos de Eucalyptus.

Considerando a serapilheira total para os três povoamentos, em geral, a ordem de aporte de nutrientes foi $\mathrm{Ca}>\mathrm{K}>\mathrm{Mg}>\mathrm{S}>\mathrm{P}$ (Tabela 4). Corroborando, em estudo com 44 espécies nativas, Villa et al. (2016) observaram a mesma ordem de aporte de nutrientes via serapilheira, ao longo de dois anos de avaliação, em época seca e chuvosa e em diferentes espaçamentos, no Estado do RJ. Nos três povoamentos a maior deposição dos nutrientes $\mathrm{P}, \mathrm{K}, \mathrm{Ca}, \mathrm{Mg}$ e $\mathrm{S}$ no solo via serapilheira, em geral, foi verificada nas estações de verão e primavera, enquanto que a menor deposição ocorreu na estação de inverno. Este fato é atribuído a menor deposição de serapilheira no período de inverno.

A maior transferência anual dos nutrientes $P$, $\mathrm{Ca}, \mathrm{K}$ e S para o solo via serapilheira ocorreu no povoamento de dois anos, enquanto que o povoamento de três anos foi o que apresentou menor transferência. Em média, o povoamento de dois anos transferiu, respectivamente, cerca de $45 \%, 46 \%, 51 \%$, e $26 \%$ a mais de P, K, Ca e $\mathrm{S}$, em relação aos povoamentos de três anos. Para o Mg, a transferência das copas para o solo foram semelhantes nos três povoamentos (Tabela 4).

A ordem e grandeza do potencial de nutrientes a ser transferido para o solo seguiu a mesma do teor dos nutrientes do componente folhas da serapilheira. Sendo a deposição das folhas responsáveis por cerca de 99\%, 97\%, $97 \%, 96 \%$ e $98 \%$ do total dos elementos $\mathrm{P}, \mathrm{K}$, $\mathrm{Ca}, \mathrm{Mg}$ e $\mathrm{S}$, a ser transferidos ao solo, nos povoamentos de um, dois e três anos, respectivamente. Em quantidade menor, a transferência de nutrientes nos povoamentos via serapilheira pode ser atribuída aos componentes galho $>$ casca (Tabela 4). De forma similar Viera et al. (2014) encontraram em E. urophylla $\times E$. globulus a seguinte ordem de transferência: folhas > galhos finos > miscelânea > galhos grossos. Em estudo com E. dunnii, Corrêa et al.(2013) encontrou a seguinte ordem de transferência: folhas > miscelânea > galhos grossos, onde a frações folhas representou $96 \%$ do aporte anual total de macronutrientes. 
Tabela 3 - Teor de nutrientes nos componentes da serapilheira em povoamentos de Eucalyptus dunnii com diferentes idades.

Table 3 - Nutrient concentration in the litter components in Eucalyptus dunnii stands of different ages.

\begin{tabular}{|c|c|c|c|c|c|c|c|c|c|}
\hline \multirow{3}{*}{$\begin{array}{l}\text { Estações } \\
\text { do ano }\end{array}$} & \multicolumn{9}{|c|}{ Povoamentos } \\
\hline & \multicolumn{3}{|c|}{1 ano de idade } & \multicolumn{3}{|c|}{2 anos de idade } & \multicolumn{3}{|c|}{3 anos de idade } \\
\hline & Folha & Galho & Casca & Folha & Galho & Casca & Folha & Galho & Casca \\
\hline & \multicolumn{9}{|c|}{ Fósforo $\left(\mathrm{g} \mathrm{kg}^{-1}\right)$} \\
\hline Verão & $0,5 \mathrm{Ba}^{2 ; 3}$ & 0,3 Aa & $0,0 \mathrm{Ba}$ & $0,6 \mathrm{Ca}$ & $0,1 \mathrm{Ab}$ & $0,6 \mathrm{Aa}$ & $0,5 \mathrm{Ba}$ & $0,2 \mathrm{Ab}$ & $0,5 \mathrm{Aa}$ \\
\hline Outono & $0,9 \mathrm{Aa}$ & $0,2 \mathrm{Aa}$ & $0,2 \mathrm{Aa}$ & $0,9 \mathrm{Ba}$ & $0,1 \mathrm{Aa}$ & $0,2 \mathrm{Ba}$ & $0,5 \mathrm{Bb}$ & $0,1 \mathrm{Aa}$ & $0,2 \mathrm{Aa}$ \\
\hline Inverno & 1,0 Aab & $0,2 \mathrm{Aa}$ & $0,4 \mathrm{Aa}$ & $1,2 \mathrm{Aa}$ & $0,2 \mathrm{Aa}$ & $0,3 \mathrm{ABab}$ & $0,9 \mathrm{Ab}$ & $0,1 \mathrm{Aa}$ & $0,2 \mathrm{Ab}$ \\
\hline \multirow[t]{2}{*}{ Primavera } & $0,9 \mathrm{Aa}$ & $0,2 \mathrm{Aa}$ & $0,3 \mathrm{Aab}$ & $0,9 \mathrm{Aa}$ & $0,3 \mathrm{Aa}$ & $0,3 \mathrm{ABa}$ & $0,8 \mathrm{Aa}$ & 0,2 Aa & $0,2 \mathrm{Ab}$ \\
\hline & \multicolumn{9}{|c|}{ Potássio $\left(\mathrm{g} \mathrm{kg}^{-1}\right)$} \\
\hline Verão & $2,2 \mathrm{Ba}$ & 2,3 Aa & $0,0 \mathrm{Ba}$ & 2,9 Ba & 2,6 Aa & $3,6 \mathrm{Aa}$ & 2,2 Aa & 1,5 Aa & $0,2 \mathrm{Aa}$ \\
\hline Outono & $5,2 \mathrm{Aa}$ & 2,4 Aa & $1,0 \mathrm{Aa}$ & $4,7 \mathrm{Aa}$ & $3,2 \mathrm{Aa}$ & $1,2 \mathrm{Ba}$ & $3,1 \mathrm{Ab}$ & 1,9 Aa & $0,7 \mathrm{Aa}$ \\
\hline Inverno & 4,3 Aab & 2,2 Aab & $1,1 \mathrm{Aa}$ & 4,6 $\mathrm{Aa}$ & 3,6 Aa & $1,1 \mathrm{Ba}$ & $3,1 \mathrm{Ab}$ & $1,8 \mathrm{Ab}$ & $0,7 \mathrm{Aa}$ \\
\hline \multirow[t]{2}{*}{ Primavera } & $2,3 \mathrm{Ba}$ & 3,0 Aab & $0,9 \mathrm{Aa}$ & $3,2 \mathrm{ABa}$ & 3,3 Aa & $1,0 \mathrm{Ba}$ & 2,1 Aa & $1,3 \mathrm{Ab}$ & $0,5 \mathrm{Aa}$ \\
\hline & \multicolumn{9}{|c|}{ Cálcio $\left(\mathrm{g} \mathrm{kg}^{-1}\right)$} \\
\hline Verão & $16,9 \mathrm{Aa}$ & 9,9 Aa & $0,0 \mathrm{Ba}$ & 15,6 Aa & $9,2 \mathrm{Aba}$ & $2,7 \mathrm{Ca}$ & $8,8 \mathrm{Ab}$ & 6,3 Aa & $3,1 \mathrm{Aa}$ \\
\hline Outono & $13,9 \mathrm{Ba}$ & $8,0 \mathrm{Ab}$ & 3,5 Aa & $12,2 \mathrm{Ba}$ & $11,3 \mathrm{Aa}$ & $3,9 \mathrm{Ba}$ & $8,0 \mathrm{ABb}$ & $5,4 \mathrm{Ab}$ & 3,3 Aa \\
\hline Inverno & $11,6 \mathrm{Ba}$ & 8,0 Aab & $4,5 \mathrm{Aa}$ & $10,5 \mathrm{Ba}$ & $8,4 \mathrm{Ba}$ & $3,9 \mathrm{Ba}$ & $6,4 \mathrm{Bb}$ & $5,8 \mathrm{Ab}$ & 3,6 Aa \\
\hline \multirow[t]{2}{*}{ Primavera } & $12,3 \mathrm{Ba}$ & 7,7 Aab & $2,9 \mathrm{Ab}$ & $10,8 \mathrm{Ba}$ & $8,3 \mathrm{Ba}$ & 4,9 Aa & $6,7 \mathrm{ABb}$ & $5,2 \mathrm{Ab}$ & $3,9 \mathrm{Ab}$ \\
\hline & \multicolumn{9}{|c|}{ Magnésio $\left(\mathrm{g} \mathrm{kg}^{-1}\right)$} \\
\hline Verão & $0,9 \mathrm{Ca}$ & $0,7 \mathrm{Aab}$ & $0,0 \mathrm{Bb}$ & $0,6 \mathrm{Bb}$ & $0,5 \mathrm{Ab}$ & $0,4 \mathrm{Bb}$ & $1,1 \mathrm{Ba}$ & $0,8 \mathrm{Aa}$ & 1,1 Aa \\
\hline Outono & $1,5 \mathrm{Aa}$ & $0,9 \mathrm{Aa}$ & 1,0 Aab & $1,0 \mathrm{Ab}$ & $0,4 \mathrm{Ab}$ & 1,0 Aab & $1,2 \mathrm{ABb}$ & 0,6 Aab & $1,2 \mathrm{Aa}$ \\
\hline Inverno & $1,3 \mathrm{Ba}$ & 0,7 Aab & 1,1 Aab & $1,0 \mathrm{Ab}$ & $0,5 \mathrm{Ab}$ & 1,1 Aab & $1,2 \mathrm{ABa}$ & $0,8 \mathrm{Aa}$ & 1,2 Aa \\
\hline \multirow[t]{2}{*}{ Primavera } & $1,1 \mathrm{BCb}$ & 0,7 Aa & $0,7 \mathrm{Aab}$ & $0,8 \mathrm{Abc}$ & $0,6 \mathrm{Aa}$ & $0,7 \mathrm{ABab}$ & $1,4 \mathrm{Aa}$ & $0,8 \mathrm{Aa}$ & 0,9 Аa \\
\hline & \multicolumn{9}{|c|}{ Enxofre $\left(\mathrm{g} \mathrm{kg}^{-1}\right)$} \\
\hline Verão & $0,9 \mathrm{Ba}$ & $0,4 \mathrm{Aa}$ & $0,0 \mathrm{Cb}$ & $0,8 \mathrm{Cb}$ & $0,3 \mathrm{Ab}$ & $0,3 \mathrm{Ba}$ & $0,7 \mathrm{Bc}$ & $0,3 \mathrm{Ab}$ & $0,3 \mathrm{Aa}$ \\
\hline Outono & $1,1 \mathrm{ABa}$ & $0,3 \mathrm{Ba}$ & $0,3 \mathrm{Ba}$ & $0,9 \mathrm{BCa}$ & $0,3 \mathrm{Aa}$ & $0,3 \mathrm{Ba}$ & $0,7 \mathrm{Bb}$ & $0,2 \mathrm{Ab}$ & $0,3 \mathrm{Aab}$ \\
\hline Inverno & $1,1 \mathrm{ABa}$ & $0,3 \mathrm{Ba}$ & $0,4 \mathrm{Aa}$ & $1,1 \mathrm{ABa}$ & $0,3 \mathrm{Aa}$ & $0,3 \mathrm{Bb}$ & $1,1 \mathrm{Aa}$ & $0,2 \mathrm{Ab}$ & $0,3 \mathrm{Ab}$ \\
\hline Primavera & 1,2 Aa & $0,4 \mathrm{Aa}$ & $0,4 \mathrm{ABa}$ & 1,3 Aa & $0,3 \mathrm{Ab}$ & 0,4 Aa & $1,1 \mathrm{Ab}$ & $0,3 \mathrm{Ab}$ & $0,3 \mathrm{Ab}$ \\
\hline
\end{tabular}

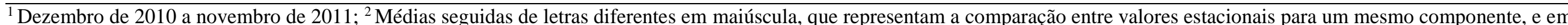

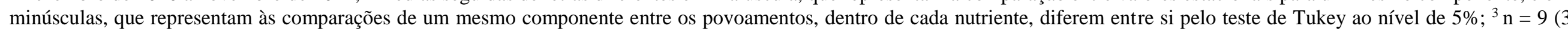
blocos x 3 meses de coleta $=$ uma estação). 
Tabela 4. Transferência de nutrientes dos componentes da serapilheira ao solo em povoamentos de Eucalyptus dunnii com diferentes idades ${ }^{1}$.

Table 4 - Deposition of nutrients by the litterfall components to soil in Eucalyptus dunnii stands of different ages.

Estações Povoamentos

\begin{tabular}{|c|c|c|c|c|c|c|c|c|c|c|c|c|}
\hline \multirow{3}{*}{$\begin{array}{l}\text { Estações } \\
\text { do ano }{ }^{2}\end{array}$} & \multicolumn{12}{|c|}{ Povoamentos } \\
\hline & \multicolumn{4}{|c|}{1 ano de idade } & \multicolumn{3}{|c|}{2 anos de idade } & \multicolumn{5}{|c|}{3 anos de idade } \\
\hline & Folha & Galho & Casca & & Folha & Galho & Casca & & Folha & Galho & Casca & \\
\hline & \multicolumn{12}{|c|}{ Fósforo $\left(\mathrm{kg} \mathrm{ha}^{-1}\right)$} \\
\hline Verão & $1,071 \mathrm{Bb}^{3}$ & $0,001 \mathrm{Aa}$ & $0,000 \mathrm{Cb}^{5}$ & & $1,341 \mathrm{Ca}$ & $0,002 \mathrm{Ba}$ & $0,001 \mathrm{Bb}$ & & $1.096 \mathrm{Bb}$ & $0,005 \mathrm{Ba}$ & $0,016 \mathrm{Ba}$ & \\
\hline Outono & $1,253 \mathrm{Ab}$ & $0,007 \mathrm{Aa}$ & $0,002 \mathrm{Bc}$ & & $1,475 \mathrm{Ba}$ & $0,004 \mathrm{Ba}$ & $0,015 \mathrm{Ab}$ & & $0,580 \mathrm{Cc}$ & $0,008 \mathrm{Ba}$ & $0,021 \mathrm{Aa}$ & \\
\hline Inverno & $1,113 \mathrm{Bb}$ & $0,006 \mathrm{Ab}$ & $0,004 \mathrm{Ab}$ & & 1,162 Da & $0,021 \mathrm{Aa}$ & 0,014 Aa & & $0,541 \mathrm{Cc}$ & $0,009 \mathrm{Bb}$ & $0,012 \mathrm{Ca}$ & \\
\hline Primavera & $1,288 \mathrm{Ab}$ & $0,020 \mathrm{Ab}$ & $0,001 \mathrm{Bc}$ & & 1,991 Aa & $0,017 \mathrm{Aa}$ & $0,003 \mathrm{Bb}$ & & $1,264 \mathrm{Ab}$ & $0,025 \mathrm{Aa}$ & $0,005 \mathrm{Da}$ & \\
\hline \multirow[t]{2}{*}{ Total } & $\begin{array}{r}4,724 b^{6} \\
(99,4 \%)^{4}\end{array}$ & $\begin{array}{l}0,034 \mathrm{c} \\
(0,4 \%)\end{array}$ & $\begin{array}{l}0,007 \mathrm{c} \\
(0,1 \%)\end{array}$ & $4,76 b^{7}$ & $\begin{array}{l}5,969 a \\
(98,8 \%)\end{array}$ & $\begin{array}{l}0,044 \mathrm{~b} \\
(0,7 \%) \\
\end{array}$ & $\begin{array}{l}0,033 \mathrm{~b} \\
(0,5 \%) \\
\end{array}$ & $6,04 \mathrm{a}$ & $\begin{array}{c}3,481 \mathrm{c} \\
(97,4 \%)\end{array}$ & $\begin{array}{l}0,047 a \\
(1,2 \%) \\
\end{array}$ & $\begin{array}{l}0,054 a \\
(1,4 \%)\end{array}$ & $3,58 \mathrm{c}$ \\
\hline & \multicolumn{12}{|c|}{ Potássio $\left(\mathrm{kg} \mathrm{ha}^{-1}\right)$} \\
\hline Verão & $4,710 \mathrm{Bb}$ & $0,007 \mathrm{Cc}$ & $0,000 \mathrm{Cc}$ & & $6,484 \mathrm{Ba}$ & $0,055 \mathrm{Db}$ & $0,004 \mathrm{Db}$ & & $4,822 \mathrm{Ab}$ & $0,039 \mathrm{Bb}$ & $0,016 \mathrm{Ca}$ & \\
\hline Outono & $7,238 \mathrm{Ab}$ & $0,089 \mathrm{Ac}$ & $0,008 \mathrm{Bc}$ & & 7,703 $\mathrm{Aa}$ & $0,141 \mathrm{Bb}$ & $0,089 \mathrm{Aa}$ & & $3,593 \mathrm{Bc}$ & $0,160 \mathrm{Aa}$ & $0,073 \mathrm{Ab}$ & \\
\hline Inverno & 4,691 Ba & $0,057 \mathrm{Bc}$ & $0,012 \mathrm{Ac}$ & & $4,457 \mathrm{Cb}$ & $0,392 \mathrm{Aa}$ & $0,051 \mathrm{Ba}$ & & $1,863 \mathrm{Cc}$ & $0,166 \mathrm{Ab}$ & $0,041 \mathrm{Bb}$ & \\
\hline Primavera & $3,291 \mathrm{Cb}$ & $0,099 \mathrm{Ab}$ & $0,001 \mathrm{Cc}$ & & $7,082 \mathrm{Ba}$ & $0,182 \mathrm{Ba}$ & $0,009 \mathrm{Cb}$ & & $3,318 \mathrm{Bb}$ & $0,160 \mathrm{Aa}$ & $0,013 \mathrm{Ca}$ & \\
\hline \multirow[t]{2}{*}{ Total } & $\begin{array}{c}19,931 \mathrm{~b} \\
(98,8 \%)^{4}\end{array}$ & $\begin{array}{l}0,252 \mathrm{a} \\
(1,1 \%)\end{array}$ & $\begin{array}{l}0,022 b \\
(0,1 \%)\end{array}$ & $20,20 \mathrm{~b}$ & $\begin{array}{l}25,726 a \\
(98,8 \%) \\
\end{array}$ & $\begin{array}{l}0,770 \mathrm{a} \\
(2,5 \%) \\
\end{array}$ & $\begin{array}{l}0,152 \mathrm{a} \\
(0,5 \%) \\
\end{array}$ & $26,64 a$ & $\begin{array}{l}13,596 \mathrm{c} \\
(95,6 \%) \\
\end{array}$ & $\begin{array}{l}0,525 \mathrm{~b} \\
(3,5 \%) \\
\end{array}$ & $\begin{array}{l}0,133 a \\
(0,9 \%)\end{array}$ & $14,25 \mathrm{c}$ \\
\hline & \multicolumn{12}{|c|}{ Cálcio $\left(\mathrm{kg} \mathrm{ha}^{-1}\right)$} \\
\hline Verão & $36,18 \mathrm{Aa}$ & $0,030 \mathrm{Cc}$ & $0,000 \mathrm{Db}$ & & $38,88 \mathrm{Ab}$ & $0,193 \mathrm{Ca}$ & $0,003 \mathrm{Db}$ & & $19,29 \mathrm{Ac}$ & $0,164 \mathrm{Db}$ & 0,099 CDa & \\
\hline Outono & $19,34 \mathrm{Ba}$ & $0,296 \mathrm{Ab}$ & $0,028 \mathrm{Bc}$ & & $19,99 \mathrm{Ca}$ & $0,497 \mathrm{Ba}$ & $0,289 \mathrm{Ab}$ & & $9,27 \mathrm{Bb}$ & $0,454 \mathrm{Ca}$ & $0,343 \mathrm{Aa}$ & \\
\hline Inverno & $12,91 \mathrm{Ca}$ & $0,208 \mathrm{Bc}$ & $0,050 \mathrm{Ac}$ & & $10,17 \mathrm{Db}$ & $0,916 \mathrm{Aa}$ & $0,179 \mathrm{Bb}$ & & $3,84 \mathrm{Cc}$ & $0,534 \mathrm{Bb}$ & $0,212 \mathrm{Ba}$ & \\
\hline Primavera & $17,60 \mathrm{Bb}$ & $0,254 \mathrm{Ac}$ & $0,003 \mathrm{Cc}$ & & $23,90 \mathrm{Ba}$ & $0,457 \mathrm{Bb}$ & $0,044 \mathrm{Cb}$ & & $10,58 \mathrm{Bc}$ & $0,640 \mathrm{Aa}$ & $0,101 \mathrm{Ca}$ & \\
\hline \multirow[t]{2}{*}{ Total } & $\begin{array}{l}86,04 a \\
(99,0 \%)^{4}\end{array}$ & $\begin{array}{l}0,788 \mathrm{c} \\
(0,9 \%)\end{array}$ & $\begin{array}{l}0,071 \mathrm{a} \\
(0,1 \%)\end{array}$ & $86,89 \mathrm{a}$ & $\begin{array}{l}88,953 \mathrm{a} \\
(97,3 \%)\end{array}$ & $\begin{array}{l}2,063 a \\
(2,2 \%) \\
\end{array}$ & $\begin{array}{l}0,515 \mathrm{a} \\
(0,5 \%) \\
\end{array}$ & $91,53 \mathrm{a}$ & $\begin{array}{l}42,994 b \\
(94,4 \%)\end{array}$ & $\begin{array}{l}1,792 \mathrm{~b} \\
(3,9 \%) \\
\end{array}$ & $\begin{array}{l}0,755 a \\
(1,7 \%)\end{array}$ & $45,54 \mathrm{~b}$ \\
\hline & \multicolumn{12}{|c|}{ Magnésio $\left(\mathrm{kg} \mathrm{ha}^{-1}\right)$} \\
\hline Verão & $1,927 \mathrm{Ab}$ & $0,002 \mathrm{Cb}$ & $0,000 \mathrm{Cb}$ & & $1,342 \mathrm{Bc}$ & $0,011 \mathrm{Cb}$ & $0,001 \mathrm{Db}$ & & $2,11 \mathrm{Aa}$ & $0,021 \mathrm{Ca}$ & $0,035 \mathrm{Ca}$ & \\
\hline Outono & $2,088 \mathrm{Aa}$ & $0,033 \mathrm{Ab}$ & $0,008 \mathrm{Bc}$ & & $1,639 \mathrm{Ab}$ & $0,018 \mathrm{Cb}$ & $0,074 \mathrm{Ab}$ & & $1,391 \mathrm{Bc}$ & $0,050 \mathrm{Ba}$ & $0,125 \mathrm{Aa}$ & \\
\hline Inverno & $1,447 \mathrm{Ba}$ & $0,018 \mathrm{Bb}$ & $0,012 \mathrm{Ac}$ & & $0,969 \mathrm{Cb}$ & $0,055 \mathrm{Aa}$ & $0,051 \mathrm{Bb}$ & & $0,721 \mathrm{Cc}$ & $0,04 \mathrm{Ba}$ & $0,071 \mathrm{Ba}$ & \\
\hline Primavera & $1,574 \mathrm{Bc}$ & $0,023 \mathrm{Bb}$ & $0,001 \mathrm{Cc}$ & & $1,770 \mathrm{Ab}$ & $0,033 \mathrm{Bb}$ & $0,006 \mathrm{Cb}$ & & $2,212 \mathrm{Aa}$ & $0,098 \mathrm{Aa}$ & $0,1023 \mathrm{Ca}$ & \\
\hline \multirow[t]{2}{*}{ Total } & $\begin{array}{l}7,036 \mathrm{a} \\
(98,6 \%)^{4}\end{array}$ & $\begin{array}{l}0,076 a \\
(1,1 \%) \\
\end{array}$ & $\begin{array}{l}0,021 \mathrm{a} \\
(0,3 \%) \\
\end{array}$ & $7,13 \mathrm{a}$ & $\begin{array}{l}5,720 \mathrm{a} \\
(96,0 \%) \\
\end{array}$ & $\begin{array}{l}0,116 a \\
(1,9 \%) \\
\end{array}$ & $\begin{array}{l}0,132 \mathrm{a} \\
(2,1 \%) \\
\end{array}$ & $5,968 \mathrm{~b}$ & $\begin{array}{l}42,994 b \\
(93,1 \%) \\
\end{array}$ & $\begin{array}{l}1,792 \mathrm{~b} \\
(3,4 \%) \\
\end{array}$ & $\begin{array}{l}0,254 \mathrm{a} \\
(3,5 \%) \\
\end{array}$ & $7,23 \mathrm{a}$ \\
\hline & \multicolumn{12}{|c|}{ Enxofre $\left(\mathrm{kg} \mathrm{ha}^{-1}\right)$} \\
\hline Verão & $1,927 \mathrm{Aa}$ & $0,001 \mathrm{Bb}$ & $0,000 \mathrm{Bb}$ & & $1,789 \mathrm{Bb}$ & $0,006 \mathrm{Ba}$ & $0,001 \mathrm{Bb}$ & & $1,534 \mathrm{Ac}$ & $0,008 \mathrm{Ba}$ & $0,001 \mathrm{Ba}$ & \\
\hline Outono & $1,531 \mathrm{Ba}$ & $0,011 \mathrm{Ab}$ & $0,028 \mathrm{Ab}$ & & $1,475 \mathrm{Ca}$ & $0,013 \mathrm{Bb}$ & 0,022 Aa & & $0,811 \mathrm{Bb}$ & $0,017 \mathrm{Ba}$ & $0,031 \mathrm{Aa}$ & \\
\hline Inverno & $1,224 \mathrm{Ca}$ & $0,008 \mathrm{Ab}$ & $0,004 \mathrm{Ab}$ & & $1,066 \mathrm{Db}$ & $0,032 \mathrm{Aa}$ & $0,014 \mathrm{Bb}$ & & $0,661 \mathrm{Cc}$ & $0,018 \mathrm{Bb}$ & 0,018 Aa & \\
\hline Primavera & $1,717 \mathrm{Bb}$ & $0,013 \mathrm{Ab}$ & $0,001 \mathrm{Ba}$ & & $2,434 \mathrm{Aa}$ & $0,016 \mathrm{Bb}$ & $0,004 \mathrm{Ba}$ & & $1,738 \mathrm{Ab}$ & $0,037 \mathrm{Aa}$ & $0,008 \mathrm{Ba}$ & \\
\hline Total & $\begin{array}{l}6,399 a \\
(99,4 \%)^{4}\end{array}$ & $\begin{array}{l}0,033 \mathrm{a} \\
(0,5 \%)\end{array}$ & $\begin{array}{l}0,007 \mathrm{~b} \\
(0,1 \%)\end{array}$ & $6,43 \mathrm{a}$ & $\begin{array}{l}6,76 a \\
(98,4 \%)\end{array}$ & $\begin{array}{l}0,061 \mathrm{a} \\
(1,0 \%)\end{array}$ & $\begin{array}{l}0,041 \mathrm{a} \\
(0,6 \%)\end{array}$ & $7.32 \mathrm{a}$ & $\begin{array}{l}4,744 a \\
(97,0 \%)\end{array}$ & $\begin{array}{l}0,080 \mathrm{a} \\
(1,6 \%) \\
\end{array}$ & $\begin{array}{l}0,058 \mathrm{a} \\
(1,4 \%)\end{array}$ & $4,81 \mathrm{~b}$ \\
\hline
\end{tabular}

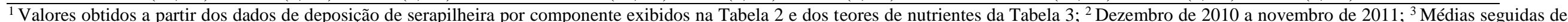

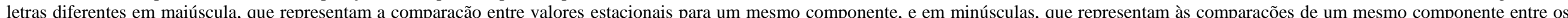

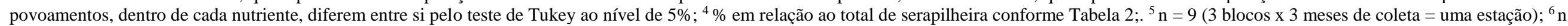
$=36$ ( 3 blocos x 3 meses de coleta x 4 estações $) ;{ }^{7} \mathrm{n}=108$ ( 3 blocos x 3 meses de coleta $\times 4$ estações x 3 componentes). 
Embora a fração folha representar um pequeno percentual em relação à biomassa total das árvores, o elevado teor de nutrientes na fração folha da serapilheira mostra o grande potencial para a ciclagem de elementos minerais, (OLADOYE et al., 2010). Desse modo, as folhas da serapilheira são responsáveis pelo retorno da maior quantidade de nutrientes ao solo em relação aos demais componentes da serapilheira (SHAMMAS, et al., 2003; OLADOYE et al., 2010).

\section{Conclusões}

Os povoamentos de um, dois e três anos produziram quantidades totais de serapilheira semelhantes, com produção média de $6.560 \mathrm{~kg}$ $\mathrm{ha}^{-1}$ ano $^{-1}$, sendo que as folhas representaram cerca de $95 \%$ da produção total.

A produção de serapilheira foi sazonal, com menor produção durante o inverno e maior no verão, quando a temperatura média mensal estava em elevação.

As folhas apresentaram maior teor de nutrientes, cuja ordem decrescente do teor foi: $\mathrm{Ca}>\mathrm{K}>\mathrm{Mg}>\mathrm{S}>\mathrm{P}$.

Tendo em vista as quantidades de nutrientes transferidas via serapilheira observadas neste estudo, se constata a importância da mesma no ciclo biogeoquímico dos nutrientes, contribuindo de maneira significativa para a manutenção da capacidade produtiva do solo, principalmente nas condições de solos de baixa fertilidade.

\section{Referências}

ASHAGRIE, Y.; ZECH, W. Litter production and nutrient cycling in two plantations and a Podocarpus falcatus dominated natural forest ecosystems in south-eastern highlands of Ethiopia Yeshanew African. Journal of Agricultural, v.8, n.38, p. 4810-4818, 2013.

BARLOW, J. et al. Litter fall and decomposition in primary, secondary and plantation forests in the Brazilian Amazon. Forest Ecology and Management, v.247, n.1-3, p.91-97, 2007.

BRAY, R. J.; GORHAM, E. Litter production in forests of the world. Advances in Ecological Research, v. 2, p.101-157, 1964.

CIZUNGU, L. et al. Litterfall and leaf litter decomposition in a central African tropical mountain forest and Eucalyptus plantation. Forest Ecology and Management, v. 326, n. 15, p.109-116, 2014.

CORRÊA, R. S.; SCHUMACHER, M. V.; MOMOLLI, D. R. Deposição de serapilheira e macronutrientes em povoamento de Eucalyptus dunnii Maiden sobre pastagem natural degradada no Bioma Pampa. Scientia Forestalis, v. 41, n. 97, p. 065-074, 2013.

CORREIA, M. E. F; ANDRADE, A. G. Formação de serapilheira e ciclagem de nutrientes. In: SANTOS, G.A.; CAMARGO, F.A. (Ed.). Fundamentos da matéria orgânica do solo: ecossistemas tropicais e subtropicais. Porto Alegre: Gênesis, 1999. p.209-214.

CUNHA NETO, F. V.et al. Acúmulo e decomposição da serapilheira em quatro formações florestais. Ciência Florestal, v. 23, n. 3, p. 379-387, 2013.

DINIZ, A. R.; PEREIRA, M. G.; LOSS, A. Aporte de material decíduo e fertilidade do solo em plantio de eucalipto e floresta secundária. Pesquisa Florestal Brasileira, v. 31, p. 19-26, 2011.

EMBRAPA - Empresa Brasileira de Pesquisa Agropecuária -. Centro Nacional de Pesquisa de Solos. Solos do Estado de Santa Catarina. Rio de Janeiro, 2004. 726p. (Boletim de Pesquisa e Desenvolvimento, 46).

EPAGRI - Empresa de Pesquisa Agropecuária e Extensão Rural de Santa Catarina. Solicitação de 
dados meteorológicos. 2012. Disponível em: <http://ciram.epagri.sc.gov.br/index.php?option =com_content \&view=article\&id=2037> .

Acesso em: 31 dez. de 2012.

FERREIRA, D. F. Programa SISVAR. Sistema de análise de variância: versão 4,6 (Build 6,0). Lavras: DEX/UFLA, 2003.

FOELKEL, C. Minerais e nutrientes das árvores dos eucaliptos: Aspectos ambientais, fisiológicos, silviculturais e industriais acerca dos elementos inorgânicos presentes nas árvores. Eucalyptus Online Book \& Newsletter. Cap. 02. Disponível em: <http://www.eucalyptus.com.br/capitulos/capit ulo_minerais.pdf $>$. Acesso em: 02 jul. de 2015.

FORTES, F. O.; DAL'COL LÚCIO, A.; STOR, L. Plano amostral para coleta de serapilheira na Floresta Ombrófila Mista do Rio Grande do Sul. Ciência Rural, v. 38, n. 9, p. 2512-2518, 2008.

FREITAS, E. C. S. et al. Deposição de serapilheira e de nutrientes no solo em Sistema Agrossilvipastoril com eucalipto e acácia. Revista Árvore, v. 37, n. 3, p. 409-417, 2013.

HERNÁNDEZ, J. et al. Nutrient export and harvest residue decomposition patterns of a Eucalyptus dunnii Maiden plantation in temperate climate of Uruguay. Forest Ecology and Management, Amsterdam, v. 258, n. 2, p. 92-99, 2009.

INKOTTE, J. et al. Deposição de serapilheira em reflorestamentos de eucalipto e florestas nativas nas regiões Planalto e Oeste do Estado de Santa Catarina, Scientia Forestalis, v. 43, n. 106, 2015.

LACLAU, J. P. et al. Biogeochemical cycles of nutrients in tropical Eucalyptus plantations: main features shown by intensive monitoring in Congo and Brazil. Forest Ecology and Management, v. 259, n. 9, p. 1771-1785, 2010.

MALUF, J. R. T. Nova classificação climática do Estado do Rio Grande do Sul. Revista
Brasileira de Agrometeorologia, v. 8, n. 1, p. 141-150, 2000.

OLADOYE, A. O.; OLA-ADAMS, B. A.; ADEDIRE, M. O. Litterfall dynamics in Leucaena leucocephala (Lam) de wit plantation in the Nigerian derived savanna. Journal of Agricultural and Biological Science, v. 5, n. 2, p. 31-38, 2010.

REIS, M. G. F.; BARROS, N. F. Ciclagem de Nutrientes em Plantios de Eucalipto. In: Barros, N. F. \& Novais, R.F. (Eds.). Relação soloeucalipto. Viçosa: Folha de Viçosa, 1990. p. 265-302.

SALVADOR, S. M; CONSENSA, C. B.; ARAÚJO, E. F. Produção de serapilheira e devolução de macronutrientes em um povoamento de Eucalyptus saligna (F. Muell). Ecologia e Nutrição Florestal, v. 2, n. 2, p. 5262, 2014.

SANTOS, H. G. et al. (Ed.). Sistema brasileiro de classificação de solos. 2.ed. Rio de janeiro: Embrapa Solos, 2006. 306p.

SCHUMACHER, M. V. et al. Espécies predominantes na deposição de serapilheira em fragmento de Floresta Estacional Decidual no Rio Grande do Sul. Ciência Florestal, v. 21, n. 3, p. 479-486, 2011.

SCHUMACHER, M. V. et al. Produção e decomposição de serapilheira em um povoamento de Eucalyptus urophylla $\times$ Eucalyptus globulus Maidenii. Cerne, v. 19, n. 3, p. 509-508, 2013.

SCHUMACHER, M. V. et al. Retorno de nutrientes via deposição de serapilheira em um povoamento de acácia-negra (Acacia mearnsii De Wild.) no Estado do Rio Grande do Sul. Revista Árvore, v. 27, n. 6, p. 791-798, 2003.

SCHUMACHER, M. V.; VIEIRA, M.; WITSCHORECK, R. Produção de serapilheira e transferência e nutrientes em área de segunda 
rotação com floresta de Pinus taeda L. no município de Cambará do Sul, RS. Ciência Florestal, v. 18, n. 4, p. 471-480, 2008.

SELLE, G. L. Ciclagem de nutrientes em ecossistemas florestais. Bioscience Journal, v. 23, n. 4, p.29-39, 2007.

SHAMMAS, K. et al. Contribution of decomposing harvest residues to nutrient cycling in a second rotation Eucalyptus globulus plantation in south - Western Australia. Biology and Fertility of Soils, v. 38, n. 4, p. 228-235, 2003.

SILVA, P. E. M. et al. Soil water dynamics and litter production in Eucalyptus and native vegetation in southeastern Brazil. Scientia Agricola, v. 71, n. 5, p.374-379, 2014.

TEDESCO, M. J. et al. Análise de solo, plantas e outros materiais. 2.ed. Porto Alegre: Departamento de Solos, UFRGS, 1995. 174p. (Boletim Técnico, 5).

TURNER, J.; LAMBERT, M. J. Nutrient cycling within a 27-years-old Eucalyptus grandis plantation in New South Wales. Forest Ecology and Management, v. 6, n. 2, p. 155-168, 1983.

VEZZANI, F. M.; MIELNICZUK, J. O solo como sistema. Curitiba: Edição dos Autores, 2011. 104 p.

VIERA, M. SCHUMACHER, M. V.; ARAÚJO, E. F.; CORRÊA, R. S.; CALDEIRA, M. V. W. Deposição de serapilheira e nutrientes em plantio de Eucalyptus urophylla $\times$ E. globulus . Floresta Ambiente, v. 21, n. 3, p. 327-338, 2014.

VIERA, M.; SCHUMACHER, M. V. Teores e aporte de nutrientes na serapilheira de Pinus taeda L., e sua relação com a temperatura do ar e pluviosidade. Revista Árvore, v. 34, n. 1, p. 8594, 2010.

VILLA, E. B. et al. Aporte de Serapilheira e
Nutrientes em Área de Restauração Florestal com Diferentes Espaçamentos de Plantio. Aporte de serapilheira e nutrientes em área de restauração florestal com diferentes espaçamentos de plantio. Floresta e Ambiente, v. 23, n. 1, p. 90-99, 2016.

VITOUSEK, P. M. Litterfall, nutrient cycling and nutrients in tropical forests. Ecology, v. 65, n. 1, p. 285-298, 1984. 